\title{
LA INVERSIÓN EN PROGRAMAS SOCIALES SELECTIVOS: PROPUESTA DE UNA HERRAMIENTA METODOLÓGICA PARA EL ANÁLISIS DE SU SOSTENIBILIDAD ${ }^{1}$
}

INVESTMENT IN SELECTIVE SOCIAL PROGRAMS: A PROPOSED METHODOLOGICAL TOOL FOR THE ANALYSIS OF PROGRAMS' SUSTAINABILITY

\author{
Manuel Antonio Barahona Montero \\ Ana Cristina Carmona Segnini ${ }^{3}$ \\ Greivin Salazar Álvarez ${ }^{4}$ \\ Joan Pamela Pan Sanabria 5
}

\begin{abstract}
Resumen
El presente artículo desarrolla una propuesta metodológica para evaluar la sostenibilidad de los Programas Sociales Selectivos (PSS), en donde se toma como base la relación entre crecimiento económico y desarrollo humano planteada por el Programa de las Naciones Unidas para el Desarrollo (PNUD, 1996). Para tales fines se elabora el Círculo de la Sostenibilidad que está integrado por un total de 12 pilares. Cada uno de estos es evaluado a partir de su estado actual e impacto, la combinación de ambos resultados no solo permite evaluar la sostenibilidad como tal de estos programas, sino que determina cuáles son las áreas de atención prioritaria. Por tanto, esta lógica facilita canalizar de mejor manera los esfuerzos y recursos disponibles.
\end{abstract}

doi: http://dx.doi.org/10.15359/eys.19-46.2

Fecha de recepción: 19-05-2014. Fechas de reenvíos: 25-07-2014/04-08-14/13-08-2014/19-08-14/11-11-2014/2411-2014. Fecha de aceptación: 11-12-2014. Fecha de publicación: 15-12-2014

${ }^{1}$ Proyecto de investigación 0492-10: Sostenibilidad de la inversión social pública en programas sociales selectivos, Escuela de Economía, Universidad Nacional, Costa Rica.

${ }^{2}$ Catedrático, Escuela de Economía, Universidad Nacional. Costa Rica. mbarahona.montero@gmail.com

${ }^{3}$ Docente e Investigadora, Escuela de Economía, Universidad Nacional. Costa Rica. cris18@racsa.co.cr

${ }^{4}$ Académico, Escuela de Economía, Universidad Nacional. Costa Rica. grsala1@gmail.com

${ }^{5}$ Asistente de Investigación, Escuela de Economía, Universidad Nacional. Costa Rica. jpps08@outlook.com

Manuel Barahona, Ana Cristina Carmona, Greivin Salazar y Joan Pamela Pan

Artículo protegido por licencia Creative Commons 
Palabras claves: Inversión social; programas sociales selectivos; sostenibilidad; política social; análisis social, herramienta metodológica.

\begin{abstract}
This paper proposes a methodology to evaluate sustainability of Selective Social Programs (SSP), based on the relationship between economic growth and human development posed by the United Nations Development Program (UNDP). For such purposes, the Circle of Sustainability is developed, which is comprised of 12 pillars. Each pillar is evaluated based on its current status and impact. Combining both results allows to assesses sustainability of these programs and identify areas of focus. Therefore, this methodology helps to better channel available efforts and resources.
\end{abstract}

Keywords: Social investment; selective social programs; sustainability; social policy; social analysis; methodological tool.

\title{
Introducción
}

El presente artículo tiene como objetivo desarrollar una alternativa metodológica para determinar la sostenibilidad de la inversión social pública en los programas sociales selectivos en Costa Rica durante la última década, sustentada en el enfoque del Programa de las Naciones unidas para el Desarrollo (PNUD) acerca de las interacciones entre crecimiento económico y desarrollo humano, para de esta manera definir las áreas de atención prioritarias en aras de mejorar la efectividad y eficiencia de dichos recursos. Esta propuesta busca trascender aquellos enfoques que concentran su análisis, primordialmente en los aspectos microeconómicos y presupuestarios de las instituciones que participan en este tipo de programas, esto al incluir elementos de carácter macro, meso y meta.

Para tales fines, el documento está integrado por tres secciones adicionales a la presente introducción, en la primera se desarrolla el marco conceptual que permite establecer qué condiciones deberían cumplirse para que la inversión en programas sociales sea sostenible: el denominado Círculo de la Sostenibilidad; en la segunda se muestra la herramienta que se ha elaborado para establecer el grado de sostenibilidad de esta inversión, así como las áreas de atención prioritarias; mientras que en la tercera y última se presentan las reflexiones derivadas de esta iniciativa. 
El artículo centra la atención exclusivamente en la propuesta metodológica y no en los resultados de la aplicación de la herramienta. Estos forman parte de otros productos del Proyecto de Investigación en el cual tuvo lugar su desarrollo.

\section{Aspectos teóricos: El Círculo de la Sostenibilidad}

\section{¿Bajo qué circunstancias la inversión social pública es sostenible?}

La primera interrogante que se planteó fue ¿bajo qué circunstancia la Inversión Social Pública (ISP) en general es sostenible? al igual que cualquier otro tipo de inversión, su sostenibilidad depende del rédito que esta genere, así como la posibilidad de que al menos una parte de dicho retorno sea nuevamente utilizado para incrementar el stock o "capital social"; es decir, que exista una especie de "reproducción ampliada".

Ambas condiciones constituyen elementos sine quo non de la sostenibilidad, pues bien, si las políticas públicas sociales no generan ese rédito -ese plus necesario-, tarde o temprano el sector público no contará con la "base material" requerida para seguir invirtiendo en estos programas. Por otra parte, sin la "reinversión" de este retorno, el ciclo como tal se estancaría o interrumpiría, lo que le imposibilitaría al Estado la atención continua de las necesidades de la sociedad.

Por ende, cabe establecer como criterio que la sostenibilidad de la ISP depende de esa relación entre desarrollo humano ("capital social") y crecimiento económico ("base material"), así como del estado de los "pilares" existentes entre ambos elementos.

Sin embargo, la ISP también posee sus particularidades, su rédito se genera de manera progresiva y a largo plazo: los recursos invertidos en educación tardarán algunos años para que se expresen en un mejoramiento de las condiciones de vida y productividad de la población, pero aún más, para que genere un aumento en la producción nacional y de los recursos con que cuenta la Hacienda Pública. Además, este tipo de inversiones genera una serie de externalidades positivas, en otras palabras, produce efectos favorables que son disfrutados por varios beneficiarios. Estas dos condiciones hacen que el mercado por sí solo haga una provisión socialmente insuficiente de este tipo de servicios, lo cual desde luego justifica la participación del Sector Público.

Este tipo de relación fue expuesta por el $\underline{\text { PNUD, }}$, en su Informe Mundial de Desarrollo Humano correspondiente al año 1996, a través del denominado "Círculo Virtuoso entre el Crecimiento Económico y el Desarrollo Humano", mismo que plantea la existencia de una relación mutua y potencializadora entre ambos elementos. 
Según el PNUD (1996, pp.76-77, texto entre paréntesis agregado propio):

[...] (Existen) dos acciones en cadena en el ciclo crecimiento económico-desarrollo humano... En la primera se ve la forma en que el crecimiento económico contribuye al desarrollo humano, y en la segunda la forma en que el desarrollo humano contribuye al crecimiento económico. En cada caso, los vínculos son fuertes pero no son ni perfectos ni perfectamente eficientes. Pero conjuntamente pueden formar círculos de causalidad que se refuerzan y que en el mejor de los casos, puedes ayudar a un país a lograr fuertes adelantos en materia de desarrollo humano y de crecimiento económico. Pero si los vínculos son débiles o están desequilibrados, un país puede atravesar períodos de desarrollo humano desequilibrado y de crecimiento desequilibrado [...]

En consecuencia, el planteamiento del PNUD sobre el Círculo Virtuoso Crecimiento EconómicoDesarrollo Humano, constituye el referente teórico utilizado para elaborar el denominado Círculo de la Sostenibilidad de la Inversión Social en Programas Selectivos, mismo que es presentado a continuación.

\section{Del Círculo Virtuoso al Círculo de la Sostenibilidad}

El Círculo Virtuoso del PNUD muestra cuáles son los elementos que en mayor medida debe cumplir una sociedad para que exista una relación mutuamente potencializadora entre el crecimiento económico y desarrollo humano. De manera análoga, el Círculo de la Sostenibilidad presenta los aspectos que se tienen que satisfacer para la que la inversión social sea sostenible, parte de la premisa básica que la sostenibilidad de la inversión social depende en primera instancia de la existencia de una cierta base material en la sociedad, misma que es proporcionada por el crecimiento económico; pero, que si se cumplen en mayor o menor medida con ciertos elementos, casi a manera de requisitos, la inversión social puede potencializar el propio desempeño económico, en otras palabras, generar un cambio positivo en la capacidad productiva potencial-tendencial de la sociedad.

Al igual que el planteamiento del PNUD, la relación que existe entre los diferentes componentes del Círculo de la Sostenibilidad es fuerte pero no perfecta, o en otras palabras, no es completamente determinante. Lo que sí es un hecho, es que desde una visión a mediano y largo plazo, ambos aspectos, el crecimiento económico y la inversión social, deben velar por su coexistencia, para así asegurar su propia sostenibilidad.

Dado lo anterior, la segunda interrogante que se planteó fue ¿cuáles son los aspectos que garantizan la sostenibilidad de la Inversión Social? Esta situación da origen a lo que se denomina en este artículo como "pilar de la sostenibilidad" o "pilar" solamente. 
En términos generales, se definió que el Círculo de la Sostenibilidad está conformado por los siguientes doce pilares:

\section{a) Ente rector definido}

Resulta fundamental contar con un ente rector que defina el norte y los objetivos, establezca las metas y las acciones generales por seguir, resguarde el cumplimiento de las primeras y actué en caso que los resultados obtenidos se estén desviando del escenario deseado, para así cumplir efectivamente con el ciclo PHVA ${ }^{6}$. También es competencia de la rectoría velar por la no duplicidad de funciones entre las diferencias instituciones del sector, así como fomentar las relaciones de cooperación y coordinación entre estas, para así generar la mayor cantidad de sinergias posibles.

\section{b) Marco institucional}

Desde luego es necesario que exista un marco institucional, que se convierta en el "instrumento" mediante el cual el Estado implemente su política social. Lo ideal, sin caer en la medida de lo posible en una situación de duplicidad de funciones, es que existan instituciones especializadas, pero a la vez coordinadas, para la atención de grupos de interés particulares, desde luego a partir de un enfoque amplio de derechos humanos, para así tratar de cubrir las necesidades de género, minorías étnicas, infancia, adulto mayor, entre otros.

\section{c) La definición e identificación de la población objetivo}

Es necesario establecer el perfil de los beneficiarios de cada uno de los programas o iniciativas de carácter universal, dirigido a hogares en condición de pobreza, únicamente para la población adulta mayor, solo por mencionar algunos. Una vez definido dicho perfil, se requiere identificar cuáles grupos sociales o personas cumplen con las características establecidas en el mismo, lo que da como resultado la obtención de la población objetivo.

Para tales fines, lo recomendable es contar con un procedimiento o sistema, que facilite la identificación de dicha población, para que así los esfuerzos sean canalizados hacia aquellas personas que realmente lo necesitan, como por ejemplo el llamado Sistema de Información de la Población Objetivo o SIPO (actualmente Sistema Nacional de Información Social [SINAIS]). Es importante tomar en cuenta, que por lo general, los recursos disponibles por el erario público son reducidos en comparación con las necesidades que en principio debe atender, a lo anterior y en otras ocasiones, también se le unen restricciones adicionales de carácter tecnológico, cultura o institucional; por tanto, es recomendable que existan no solamente criterios para la

\footnotetext{
${ }^{6}$ El ciclo PHVA es una herramienta utilizada en los proceso de mejora continua, que fue presentada en el año 1950 por Deming y está integrada por cuatro pasos: planificar $(P)$, hacer $(H)$, verificar $(V)$ y actuar $(A)$.
} 
identificación de la población objetivo, sino también para su priorización y por ende, para la definición de la llamada población meta ${ }^{7}$.

\section{d) La eficiencia y eficacia en el suministro de bienes y servicios sociales}

Los recursos destinados a los Programas Sociales Selectivos (PSS) son canalizados hacia sus beneficiarios esencialmente de dos formas; primero, mediante el suministro de bienes 0 servicios (educación o salud por ejemplo) y segundo, a través de las ayudas complementarias al ingreso de los hogares o personas, ya sea para uso "discrecional" o bien "específico". Independientemente del canal utilizado, la eficiencia y eficacia con que se utilizan los recursos es fundamental, lo que hace indispensable contar con un sistema de indicadores básicos de desempeño, metas asociadas a estos, así como un proceso de rendición de cuentas -no como un simple hito administrativo- plenamente institucionalizado.

\section{e) Los controles en el uso de las transferencias con fines específicos}

Para los programas vinculados con las transferencias o ayudas complementarias al ingreso, es indispensable conocer qué porcentaje del presupuesto se utiliza para fines administrativos y que otro es el que finalmente llega a la población meta. Indicadores sobre la cantidad de beneficiarios o número de ayudas entregadas son importantes, pero deben ser complementados con la anterior información; en cambio, para los programas asociados al suministro de bienes o servicios, además de conocer el peso de la actividad administrativa, es importante establecer indicadores de productividad.

En el caso de las ayudas complementarias al ingreso, aparte de la eficiencia y eficacia comentada, existe un elemento más por considerar, que va a depender del enfoque del programa como tal, si este último es una transferencia monetaria no condicionada y en consecuencia, no existe un objetivo concreto, sino muy general alrededor del mismo, sus beneficiarios pueden hacer uso de esta ayuda para los fines que ellos consideren como los "más satisfactorios" $^{8}$, en consecuencia, no es necesario ni posible establecer algún tipo de control. Existe el riesgo inevitable de que estos recursos sean utilizados en actividades que no potencialicen el Círculo de la Sostenibilidad; no obstante, el sector público no debe renunciar a

\footnotetext{
${ }^{7}$ Dado lo anterior, se utilizará el término población objetivo para hacer referencia a la población que requiere de los programas de asistencia y promoción social; en cambio, la población meta indica aquella parte de la primera, que una vez evaluados las restricciones existentes, efectivamente será beneficiaria de estos programas.

${ }^{8}$ Nótese que se usa el término "más satisfactorios" en vez de "más adecuados", esto para resaltar el aspecto subjetivo que conlleva el uso y destino de estos recursos por parte de su beneficiario, mismo que se encuentra intrínseco en el primer término, a diferencia del segundo, que reviste más una valoración social sobre lo que es y no es correcto.
}

22

Manuel Barahona, Ana Cristina Carmona, Greivin Salazar y Joan Pamela Pan

Artículo protegido por licencia Creative Commons 
este tipo de iniciativas y puede recurrir a la concientización y educación ciudadana ${ }^{9}$, como mecanismo para minimizar esta fuga de recursos hacia otros fines no tan deseables.

El régimen de pensión no contributivo de la Caja Costarricense del Seguro Social (CCSS), por ejemplo, es un programa que cumple con estas características. Grosso modo, su objetivo es mejorar la calidad de vida de la persona adulta mayor que no cuenta con una pensión, mediante un aporte monetario periódico; sin embargo, la conexión entre estos dos elementos queda diluida, por la falta de especificidad en su alcance. Caso contrario, son aquellos programas donde los recursos transferidos al beneficiario tienen un objetivo específico, bajo estas circunstancias sí se requiere de algún tipo de control, para asegurar el uso adecuado de los mismos (los llamados "bonos" o "becas" son una buena muestra de este tipo de iniciativas).

\section{f) El incremento en las capacidades de las personas}

Los PSS deben facilitar o bien incidir directamente en las condiciones y calidad de vida de las personas. En este sentido, es un hecho prácticamente irrefutable que las políticas dirigidas a fortalecer el desarrollo humano también tienen un impacto positivo sobre la producción económica superando la visión del crecimiento económico como un proceso inherentemente cruel (Sen, 1998). Por ello, el incremento en las capacidades de las personas, constituye el cuarto pilar del Círculo de la Sostenibilidad.

\section{g) El incremento en la empleabilidad potencial de las personas}

El incremento de las capacidades de las personas no es un fin en sí mismo, sino que debe ser encaminado hacia la consecución de otro objetivo intermedio, como es el mejoramiento de su empleabilidad, para facilitarle su inserción en el mercado laboral, así como la obtención de un empleo de calidad, lo que se traduce en un impacto positivo sobre la economía familiar y nacional. Para que esta mejora en la empleabilidad sea efectiva, es necesaria una estrecha comunicación entre las unidades generadoras y formadoras de conocimientos -llámense universidades e institutos de educación especializada- y el tejido empresarial; en otras palabras, resulta fundamental la creación de una sinergia competitiva entre el sector público y la iniciativa privada.

Este pilar permite visualizar que el éxito de la política social requiere de la participación e interacción de diversos entes del sector público, así como de la complementariedad estratégica que puedan establecer con la iniciativa privada. En este sentido, para potencializar los efectos del mejoramiento en la empleabilidad de las personas es preciso cumplir con dos factores

\footnotetext{
${ }^{9}$ Por educación ciudadana se debe entender como todas aquellas campañas que realiza el sector público en los diferentes ámbitos de su accionar, para concientizar a la población sobre sus responsabilidades como miembro de una sociedad.
} 
adicionales, uno que comete la función de crear nuevas oportunidades a la economía (desde luego también retos) y otro que ayuda a su socialización.

\section{h) La política productiva inclusiva}

Una política productiva inclusiva corresponde al conjunto de medidas implementadas por el sector público para facilitar la incorporación de más y nuevos actores dentro de la dinámica productiva dominante, mismos que en sus condiciones normales, les sería aún más difícil. Este tipo de política puede estar integrada por una gran cantidad de medidas, sin el objetivo de ser exhaustivos, algunos de estas son la implementación de la banca de desarrollo, promoción de la pequeña y mediana empresa (PYME) y política de compras en el sector público para el fomento productivo.

\section{i) El impulso al esfuerzo exportador y de atracción de la inversión extranjera directa}

La política productiva inclusiva debe ser complementada con otro pilar, como es el impulso al esfuerzo exportador y de atracción de inversión extranjera directa (IED). Las economías latinoamericanas desde mediados de la década de los ochenta del pasado siglo -probablemente con la excepción de Chile que fue una experiencia temprana de apertura y liberalización- se dieron a la tarea de fomentar sus exportaciones, especialmente las no tradicionales y a terceros mercados, como mecanismo de generación de un mayor progreso económico.

Por tanto, dada esta orientación hacia los mercados internacionales, lo que se debe alcanzar para favorecer el desempeño económico es que:

[...] la producción de bienes que supongan un empleo intensivo de conocimientos y tecnología, así como la creación de redes productivas y de servicios articulados en torno a las exportaciones [...] (Hounie, Pittaluga, Porcile y Scatolin, 1999, p. 20).

\section{j) El incremento efectivo en la producción nacional}

De cumplirse en alguna medida los dos anteriores pilares, sin duda alguna existirá un impacto favorable sobre el crecimiento potencial de la economía, es decir un efecto multiplicador sobre la dinámica productiva nacional.

\section{k) La política macroeconómica favorable}

Debe existir un sistema tributario que traduzca el mayor dinamismo económico que genera la inversión en desarrollo humano en ingresos frescos para la hacienda pública. La velocidad con que se dé este efecto así como la cuantía del mismo va a depender de la estructura tributaria 
del país y de su capacidad de gravar a los sectores más dinámicos de la economía, claro está sin afectar su nivel de competitividad.

También es preciso, contar con ciertos grados de libertad en las finanzas públicas para poder incrementar el gasto social en general en situaciones de mayor vulnerabilidad (crisis $y$ recesiones) pero a la vez, superado este escenario, no comprometer la lógica normal de la gestión pública en esta materia.

\section{I) El compromiso político a favor de la ISP}

La mayor disponibilidad de recursos con que cuenta la Hacienda Pública, no asegura la reinversión de estos en busca del desarrollo social del país en general y de los PSS en particular. Para que esto sea realidad, es necesario contar con el apoyo político -en el sentido amplio- a favor de este tipo de programas. Es decir, que dentro de las prioridades de los diferentes actores sociales, se encuentre el fortalecimiento del desarrollo humano y que este forme parte del conjunto de aspiraciones y valores existente a nivel país. Solo de esta manera, los recursos generados por la inversión social serán nuevamente utilizados para mejorar las condiciones de vida de la población, de tal manera que se complemente el ciclo y se asegure su sostenibilidad $^{10}$.

Esta última consideración tiene sus implicaciones, primero, porque el nivel al que pertenece el pilar da señales sobre a quién puede recaer su responsabilidad y segundo, por cuanto el alcance temporal brinda una idea acerca del lapso en que se pueden obtener los resultados deseados (en el caso de un mejoramiento del círculo) o bien, del "bono social generacional ${ }^{11 "}$ existente (en el caso de un deterioro o estancamiento del círculo).

En la Tabla 1 se muestra la clasificación de los pilares según su nivel y alcance temporal:

\footnotetext{
${ }^{10}$ En Costa Rica, por ejemplo, este pilar fue fortalecido con una decisión histórica tomada durante la primera mitad del siglo anterior, cuando el 1. de diciembre de 1948, en el antiguo Cuartel Militar Bella Vista -actualmente Museo Nacional- el entonces presidente de la Junta de Gobierno, señor José Figueres Ferrer, decretó la abolición del ejército.

${ }^{11}$ Para efectos de la temática, se habla de la existencia de un "bono social generacional" cuando una sociedad disfruta de condiciones favorables de desarrollo humano, a pesar del deterioro o estancamiento de las políticas actuales para su fortalecimiento, esto debido a los efectos inerciales que aún tienen las medidas públicas anteriormente implementadas. En este caso, los logros y mejoras alcanzadas por dicha sociedad se encuentran en riesgo, en caso de no revertir la tendencia observada.
}

Manuel Barahona, Ana Cristina Carmona, Greivin Salazar y Joan Pamela Pan 
Tabla 1.

Nivel y alcance de los pilares del Círculo de la Sostenibilidad

\begin{tabular}{|c|c|c|c|c|}
\hline \multirow{2}{*}{ Alcance } & \multicolumn{4}{|c|}{ Nivel } \\
\hline & Micro & Meso & Macro & Meta \\
\hline Corto & $\begin{array}{c}\text { Definición e } \\
\text { identificación } \\
\text { población objetivo } \\
\text { controles uso } \\
\text { transferencias }\end{array}$ & -- & $\begin{array}{c}\text { Política } \\
\text { macroeconómica }\end{array}$ & -- \\
\hline Mediano & $\begin{array}{c}\text { Eficiencia y eficacia } \\
\text { del suministro }\end{array}$ & $\begin{array}{c}\text { Marco institucional y } \\
\text { rectoría } \\
\text { política productiva } \\
\text { inclusiva } \\
\text { el incremento en la } \\
\text { empleabilidad } \\
\text { potencial de las } \\
\text { personas }\end{array}$ & $\begin{array}{l}\text { Impulso al esfuerzo } \\
\text { exportador y de } \\
\text { atracción de IED }\end{array}$ & $\begin{array}{l}\text { Compromiso } \\
\text { político }\end{array}$ \\
\hline Largo & $\begin{array}{c}\text { Incremento en las } \\
\text { capacidades de las } \\
\text { personas }\end{array}$ & -- & $\begin{array}{l}\text { Incremento en la } \\
\text { producción }\end{array}$ & -- \\
\hline
\end{tabular}

Fuente: Elaboración propia.

\section{La Herramienta metodológica propuesta}

Una vez definido el Círculo de la Sostenibilidad así como sus respectivos pilares se planteó la siguiente pregunta: ¿cómo establecer áreas de atención prioritarias? Para solventar esta inquietud y en procura de respetar los principios de eficiencia y oportunidad que se le deseó dar a esta propuesta metodológica desde un inicio, se formularon dos interrogantes adicionales:

\section{¿Cuál es el estado actual del pilar? y ¿cuál es su importancia relativa?}

Nótese, que la combinación de ambas variables, es decir estado actual e importancia relativa, permite priorizar mejor las áreas de atención, en el sentido de que facilita canalizar esfuerzos y recursos a aquellas áreas que se encuentran en una situación relativa menos favorable, pero que a la vez, tienen un mayor impacto en la sostenibilidad de los PSS.

Es de esta manera, que cada uno de los pilares fue evaluado a partir de dos escalas: primero, el estado actual, en referencia a su grado de implementación o desarrollo; segundo, el impacto 
como indicador de la importancia relativa ${ }^{12}$, que tiene para la sostenibilidad de la IS. Ambos aspectos se presentan a continuación:

\section{Estado actual del pilar}

La escala utilizada para medir el estado actual del pilar varía entre 1 y 5, el valor mínimo refleja la no existencia del mismo, mientras que los otros indican diferentes grados en su implementación, tal como se detalla seguidamente: (1) no existe, (2) a nivel de concepción, (3) nivel de desarrollo de acciones-iniciales, (4) en implementación y (5) en revisión-optimización.

Para determinar el estado actual de cada uno de los pilares, la metodología propuesta plantea a manera de interrogante, una serie de parámetros de referencia. Por tanto, para realizar este ejercicio, los evaluadores deben presentar antes algún tipo de información, ya sea cuantitativa o cualitativa, que respalde la posición a externar.

En la tabla 2, se presentan dichas interrogantes así como el tipo de información a la que se puede recurrir para fundamentar la evaluación que se vaya a plantear.

Tabla 2 .

Parámetros de los pilares del Círculo de la Sostenibilidad

\begin{tabular}{|c|c|c|}
\hline Pilar & Interrogante: ¿Existe... & Información / Evidencia \\
\hline Rectoría & $\begin{array}{l}\text {... un ente rector que establezca el } \\
\text { norte, metas, objetivos y acciones de } \\
\text { las instituciones del sector? }\end{array}$ & $\begin{array}{l}\text { Identificación del ente rector del sector así como de } \\
\text { su sostenibilidad o permanencia ante los cambios de } \\
\text { Gobierno. }\end{array}$ \\
\hline $\begin{array}{l}\text { Marco } \\
\text { institucional }\end{array}$ & $\begin{array}{l}\text {... un conjunto de instituciones que } \\
\text { permiten implementar la política } \\
\text { social, basado en un enfoque amplio } \\
\text { de derechos? }\end{array}$ & $\begin{array}{l}\text { Recuento de las instituciones existentes, su año de } \\
\text { creación, marco filosófico, objetivos, campo de } \\
\text { acción. }\end{array}$ \\
\hline $\begin{array}{l}\text { Definición e } \\
\text { identificación de la } \\
\text { población } \\
\text { objetivo. }\end{array}$ & $\begin{array}{l}\ldots \text { un sistema para la identificación de } \\
\text { la población objetivo? } \\
\ldots \text { un sistema para la identificación de } \\
\text { la población meta o bien, para la } \\
\text { priorización de la población objetivo? }\end{array}$ & $\begin{array}{l}\text { Criterios utilizados para la selección de los } \\
\text { beneficiarios de los diferentes programas. } \\
\text { Balance sobre el funcionamiento del sistema } \\
\text { utilizado para identificar y priorizar la población } \\
\text { objetivo. }\end{array}$ \\
\hline $\begin{array}{l}\text { Eficiencia } \\
\text { eficacia en el } \\
\text { suministro de } \\
\text { bienes y servicios } \\
\text { sociales. }\end{array}$ & $\begin{array}{l}\text {... un sistema de indicadores de } \\
\text { desempeño, con sus respectivas } \\
\text { metas y un esquema de incentivos } \\
\text { que responda al cumplimiento de las } \\
\text { mismas? }\end{array}$ & $\begin{array}{l}\text { Identificación y balance de los sistemas de } \\
\text { indicadores de desempeño, así como de las acciones } \\
\text { derivadas a partir de los resultados obtenidos. } \\
\text { Efectividad de los procesos de rendición de cuentas } \\
\text { institucionalizados. }\end{array}$ \\
\hline & & Continúa... \\
\hline
\end{tabular}

Manuel Barahona, Ana Cristina Carmona, Greivin Salazar y Joan Pamela Pan 


\begin{tabular}{l}
\multicolumn{1}{c}{ Pilar } \\
\hline Controles en el \\
uso de las \\
transferencias con \\
fines específicos. \\
Incremento en las \\
capacidades de las \\
personas.
\end{tabular}

Incremento en la empleabilidad potencial de las personas.

Política productiva inclusiva.

Impulso al
esfuerzo
exportador y de
atracción de la
inversión
extranjera directa.

Incremento efectivo en la producción nacional.

Política macroeconómica favorable.

Compromiso político a favor de la ISP.
Interrogante: ¿Existe...

... un sistema o mecanismo para el control de las transferencias con fines específicos?

...en las últimas décadas y desde una perspectiva de desarrollo humano, un incremento sostenido en las capacidades de la sociedad de manera equilibrada?

... un vínculo entre las instituciones formadoras de conocimiento y la llamada "nueva economía", que facilite a los empleadores cubrir los puestos de trabajo requeridos?

... un mecanismo de Banca de Desarrollo?

... una política para la promoción y fomento de las MIPYMES?

... una política de compras públicas para el fomento productivo?

... una política articulada para la promoción de exportaciones, apertura de nuevos mercados y atracción de inversión extranjera directa que logre una mayor diversificación tanto de la oferta exportable como de los mercados de destino?

... una relación estrecha entre capacidad de las personas y su nivel de ingreso?

... una política macroeconómica con suficientes grados de libertad, sobretodo en el frente fiscal, que asegure de forma oportuna a las instituciones los recursos financieros necesarios?

... en la agenda de los principales actores políticos una preocupación real por el desarrollo humano, que derive en una agenda nacional relativamente consensuada?

\section{Información / Evidencia}

Desviaciones en el uso de los recursos con fines específicos.

Desempeño y resultados comparativos del país en el Índice de Desarrollo Humano (IDH) en las últimas décadas.

Resultados del IDH ajustado por la desigualdad, indicadores de pobreza y distribución del ingreso.

Resultados de las encuestas de escasez del talento humano.

Graduados en las universidades según área de conocimiento.

Balance de los programas de enseñanza del inglés e informática educativa en primaria y secundaria.

Legislación existente para la promoción efectiva de un sistema de banca de desarrollo, fomento de las MIPYMES y participación de estas en los procesos de compras públicas.

Resultados obtenidos por la implementación de este tipo de políticas.

Revisión de indicadores sobre desempeño comercial: grado de apertura económica, exportaciones e inversión extranjera directa por habitante.

Composición y concentración de las exportaciones según mercado y tipo de bien por intensidad tecnológica.

Información cuantitativa sobre el vínculo entre desarrollo humano e ingreso de las personas.

Indicadores sobre la capacidad del sistema tributario de generar ingresos frescos (elasticidad ingresosactividad productiva)

Comportamiento del déficit fiscal y su sostenibilidad a lo largo de las diferentes fases del ciclo económico

Posición de los partidos políticos, cámaras empresariales, grupos sindicales, entre otros, sobre las cuales deben ser las prioridades del gasto público social.

Fuente: Elaboración propia. 


\section{Impacto del pilar}

Por su parte, para medir el impacto de cada uno de los pilares dentro del Círculo se utilizan las siguientes valoraciones relativas ${ }^{13}$ : (1) más alto, (2) ligeramente más alto, (3) igual de alto, (4) ligeramente menos alto y (5) menos alto.

Por tanto, el anterior ejercicio se reduce a una tarea de asignación de "ponderadores" o "pesos relativos". Para tales efectos, se sugiere recurrir a un proceso deliberativo a partir del criterio de un grupo de expertos, que al final se traduzca en un consenso sobre el impacto de cada uno de los pilares.

Para el caso de la investigación de la que se deriva el presente artículo, se utilizó para dichos fines el Proceso Analítico Jerárquico ${ }^{14}$.

\section{Posibles Resultados: estado actual-impacto}

Al combinar ambas escalas (estado actual del pilar EAP e impacto del pilar IP) y establecer el valor de 3 como umbral (menor o igual que), se obtienen cuatro posibles resultados, tal como se presenta con fines ilustrativos en la figura 1:

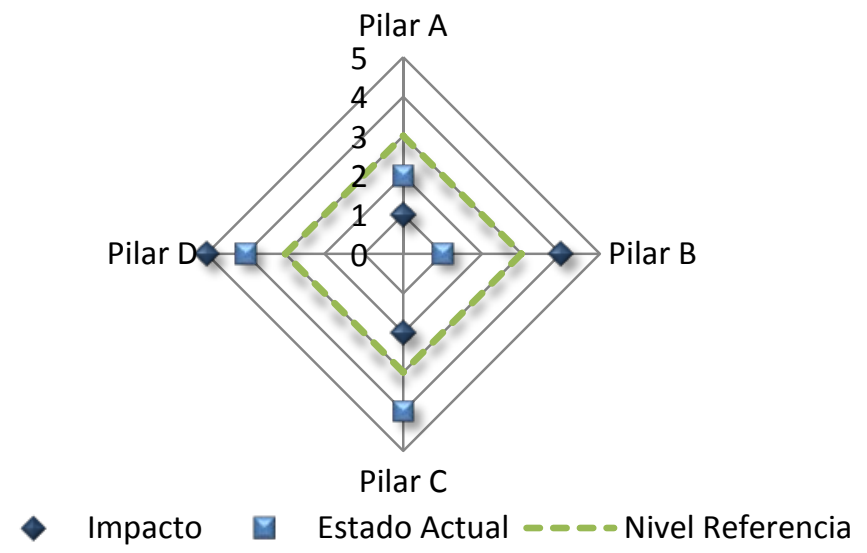

Figura 1. Resultados probables del estado actual-impacto. Fuente: Elaboración propia.

\footnotetext{
${ }^{13}$ Nótese que los calificativos utilizados se encuentran expresados en términos comparativos, esto para indicar que todos los pilares son importantes, ya que en caso contrario no tendría sentido su incorporación en el Círculo.

${ }^{14}$ A grandes rasgos, este proceso evalúa mediante una matriz cuadrada, $\mathrm{n} \times \mathrm{n}$, la importancia relativa de cada ítem con respecto a todos los demás, a partir de una escala previamente definida. Al final, una vez evaluada la consistencia de las respuestas se asigna un ponderador a cada uno de los ítems.
}

Manuel Barahona, Ana Cristina Carmona, Greivin Salazar y Joan Pamela Pan 
En este caso hipotético, los resultados obtenidos mostrarían las siguientes particularidades:

- Pilar A: el impacto es de primer orden (IP $\leq 3)$, sin embargo su estado actual indica que no está del todo desarrollado (EAP $\leq 3)$, por tanto merece una atención urgente.

- Pilar B: el impacto no es relativamente tan significativo (IP>3) y su estado actual indica que no está desarrollado (EAP $\leq 3)$, por tanto merece una atención intermedia.

- Pilar C: el impacto es de primer orden (IR $\leq 3)$ y su estado actual es satisfactorio (EAP>3), por tanto requiere de un proceso de optimización o mejora continua.

- Pilar D: el impacto no es relativamente tan significativo (IP>3) y su estado actual es satisfactorio $(\mathrm{EAP}>3)$, por tanto amerita un proceso de mantenimiento.

Dadas las anteriores opciones, podría ser que los resultados obtenidos se concentren alrededor de pilares de un tipo u otro, lo que brinda una señal sobre el grado de sostenibilidad de los PSS así como del tipo de atención requerida. En la siguiente sección se desarrolla este tema.

\section{Implicaciones de los posibles resultados}

La herramienta propuesta está diseñada para que los resultados alcanzados sobre el estado actual-impacto den señales sobre el nivel de sostenibilidad de los PSS. En este sentido, si dicho ejercicio da una concentración de los resultados en pilares tipo A, implica que existen grandes oportunidades de mejora e incluso la sostenibilidad de la IS hasta cierto punto está en riesgo, dado que se está desatendiendo aspectos con un impacto de primer orden. Bajo estas circunstancias, lo ideal es reorientar los esfuerzos para alcanzar una situación donde los resultados tiendan a agruparse en pilares tipo $B$; es decir, hacia un escenario que favorece más el desarrollo humano, ya que una vez alcanzado este punto, lo que se debe buscar es un proceso de mejora continua.

Por el contrario, si existiera un sesgo hacia pilares tipo $\mathrm{C}$ indica que en cierta medida no se ha dado una asignación eficiente de prioridades, debido a que se están atendiendo aspectos con un impacto no tan significativo. La interrogante que surge en estos casos, versa sobre la posibilidad de trasladar recursos desde aquellos pilares con un impacto no tan significativo a aquellos otros que sí lo poseen, desde luego esta alternativa requiere de una importante flexibilidad intra e interinstitucional.

La síntesis de los cuatro resultados posibles de la combinación estado actual-impacto se muestra en la figura 2 : 


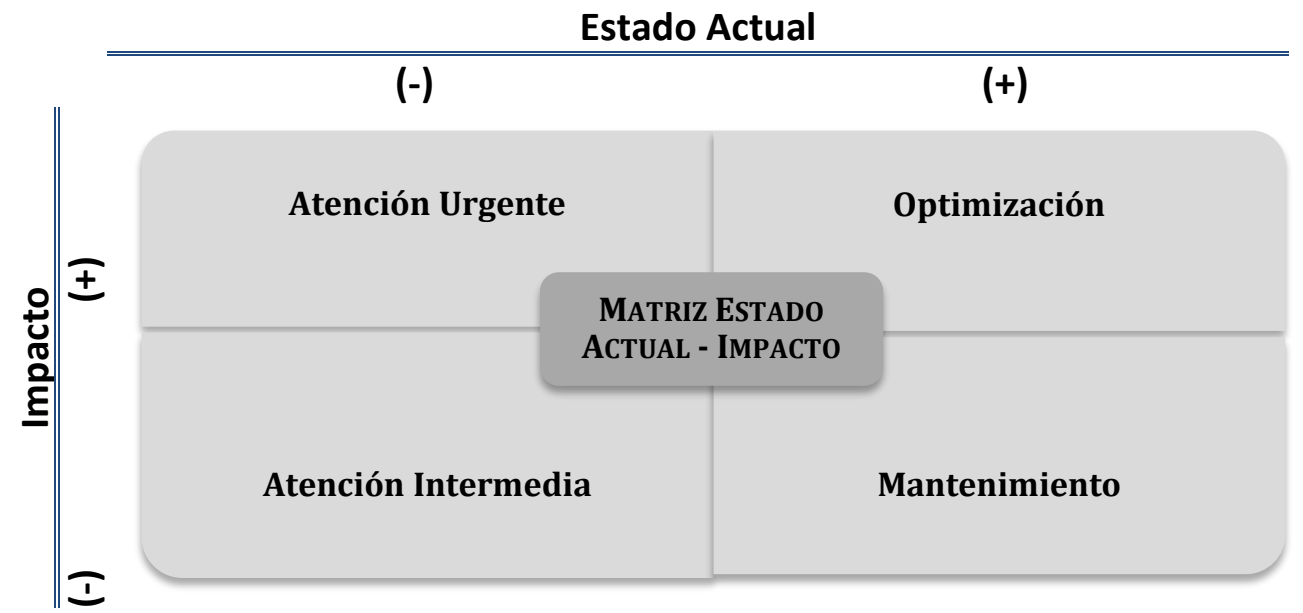

Figura 2. Matriz de resultados estado actual-impacto. Fuente: Elaboración propia.

Una vez realizado este análisis e identificado el tipo de atención que requiere cada pilar, corresponde verificar su alcance temporal. ¿Qué implicaciones tiene este cruce de información? Pues bien, se pueden obtener una serie de conclusiones adicionales, como por ejemplo qué tan urgente es tomar medidas correctivas, cuánto tiempo debe esperar la sociedad para ver alguna mejora, qué tanto margen de maniobra existe para sostener una situación relativamente favorable, entre otras.

En síntesis. La metodología propuesta se resume de la siguiente forma:

- El objetivo es articular una herramienta para evaluar la sostenibilidad de la ISP, concretamente para los PSS y definir áreas de acción prioritarias.

- Para cumplir con este objetivo, se formuló un referente teórico, denominado como el Círculo de la Sostenibilidad, basado en el enfoque del PNUD sobre crecimiento económico y desarrollo humano.

- Este referente está constituido por un total de 12 pilares, mismos que determinan el grado de sostenibilidad de la ISP.

- Estos pilares poseen un nivel (micro, meso, macro y meta) así como un alcance temporal, el primero brinda señales sobre en quien puede recaer su responsabilidad y el segundo, ofrece una idea acerca del lapso en que se puede obtener los resultados deseados o bien sostener una situación no óptima. 
- Cada uno de los pilares es evaluado en cuanto a su estado actual e impacto. Esta combinación arroja cuatro resultados posibles: atención urgente, optimización, mantenimiento, atención intermedia.

- En función de estos resultados se definen áreas de atención prioritarias, las que a su vez determinan el tratamiento que debería recibir cada pilar para fortalecer la sostenibilidad de los PSS.

\section{Reflexiones Finales}

Desde un principio, cuando se definió el objetivo general de la investigación de la cual se deriva este artículo, "evaluar la sostenibilidad de los programas sociales selectivos", se planteó el desafío de cómo abordar este estudio y a la vez separarse de aquellos enfoques que basan su análisis fundamentalmente en el tratamiento de los aspectos relacionados con el presupuesto y financiamiento de las instituciones que integran este sector.

Fue de esta manera, que se formuló el denominado Círculo de la Sostenibilidad de los Programas Sociales Selectivos como instrumento guía. Sin que se quiera ser pretencioso, se puede argumentar que este planteamiento así como la metodología para clasificar el tipo de atención y tratamiento de cada uno de los pilares que integran este Círculo, es un aporte teórico importante para el estudio, no solamente de esta temática, sino de otras relativamente similares, que involucran el uso de recursos públicos y una mutua interdependencia entre desarrollo humano y crecimiento económico.

No obstante, también existe consciencia y claridad que la evaluación del estado actual de cada uno de estos pilares, tal como la propia metodología utilizada lo exige, se podría constituir en una investigación per se, dada la complejidad y la gran cantidad de aristas que involucran los mismos, de manera tal que el esfuerzo se inscriba en el desarrollo de una agenda de investigación más amplia.

Algo similar sucede con el proceso sugerido para evaluar la importancia relativa de los pilares donde si bien es cierto que el mismo se sustenta en criterio experto cabe la introducción de ajustes y mejoras dentro de una agenda de investigación más amplia que consulte y pondere la voz de los operadores de políticas y programas sociales selectivos.

Por último, se valora como satisfactorio el desarrollo de la herramienta, ya que permite contar con una visión amplia sobre los elementos de los cuales depende la sostenibilidad de los PSS y articula un mecanismo para priorizar su atención. 


\section{Referencias}

Hounie, A., Pittaluga, L., Porcile, G. y Scatolin, F. (agosto, 1999). La CEPAL y las nuevas teorías del crecimiento. Revista de la CEPAL 68, pp. 7-33. Recuperado de: http://www.cepal.org/publicaciones/xml/2/19212/hounieesp.pdf

Programa de las Naciones Unidas para el Desarrollo- PNUD. (1996). Informe sobre Desarrollo Humano 1996. Madrid, España: Ediciones Mundi-Prensa. Recuperado de: http://biblioteca.hegoa.ehu.es/system/ebooks/3195/original/Informe sobre Desarroll o Humano 1996.pdf

Sen, A. (1998). Las teorías del desarrollo a principios del siglo XXI. En L. Emmerij y J. Núñez. (Eds.). El desarrollo económico y social en los umbrales del siglo XXI. Washington, Estados Unidos: Banco Interamericano de Desarrollo. Recuperado de: http://www.revistas.unal.edu.co/index.php/ceconomia/article/viewFile/11497/20792 